\title{
SWAP: SUN WATCHER WITH A NEW EUV TELESCOPE ON A TECHNOLOGY DEMONSTRATION PLATFORM
}

\author{
Jean-Marc Defise ${ }^{(1)}$, Jean-Hervé Lecat ${ }^{(1)}$, Emmanuel Mazy $^{(1)}$, Pierre Rochus ${ }^{(1)}$, Laurence $\operatorname{Rossi}^{(1)}$, Tanguy \\ Thibert $^{(1)}$, Jean-Marie Gillis ${ }^{(1)}$, David Berghmans ${ }^{(2)}$, Jean-François Hochedez ${ }^{(2)}$, Udo Schühle ${ }^{(3)}$ \\ ${ }^{(1)}$ Centre Spatial de Liège, av. Pré Aily 4031 Angleur, Belgium, E-mail:jmdefise@ulg.ac.be \\ ${ }^{(2)}$ Royal Observatory of Belgium, av. Circulaire, Uccle, Belgium, E-mail:david.berghmans@oma.be \\ (3) Max Planck Institut für Aeronomie, Max-Planck-Str. 2, 37191 Katlenburg-Lindau, Germany
}

\section{ABSTRACT}

SWAP (SWAP (Sun Watcher using Active Pixel System detector and Image Processing) is an instrument that has been selected to fly on the PROBA2 technology demonstration platform, a program of the European Space Agency (ESA) to be launched in 2006.

SWAP is based on an off-axis degraded Ritchey Chretien telescope that will image the EUV solar corona at $19.5 \mathrm{~nm}$ on a specifically fabricated extreme ultraviolet (EUV) sensitivity enhanced CMOS APS detector.

The optical design and the optical coatings are derived from the Extreme Ultraviolet Imaging Telescope (EIT) operating on-board SOHO since 1995 [1]. It has been adapted for a single wavelength telescope with off-axis optics. It allows to use smaller optics and filters, with simple internal baffles avoiding external protruding parts. The superpolished optics will receive a multilayer coating that provides spectral selection centred on $19.5 \mathrm{~nm}$ and EUV reflectivity in normal incidence. This compact design is specifically adapted for accommodation on PROBA-2, where mass and envelope requirements are very stringent

The SWAP PROBA-2 program will be an opportunity to demonstrate this new optical concept, while it will also validate space remote sensing with APS detectors, as well as on-board image processing capabilities.

On the science outcomes, SWAP will provide solar corona images in the Fe XII line on a baselined 2-min cadence. Observations with this specific wavelength allow detecting phenomena, such as solar flares or 'EIT-waves', associated with the early phase of coronal mass ejections. The SWAP data will complement the observations provided by SOHO-EIT, and STEREOSECCHI.

\section{THE PROBA-2 PLATFORM}

The PROBA-2 small satellite (> $120 \mathrm{~kg}$ ), to be developed under an ESA General Support Technology
Program (GSTP) contract by a consortium led by Verhaert Design \& Development (Belgium) has two main mission objectives: (i) perform an in-flight demonstration of a series of new spacecraft technologies and (ii) support a scientific mission of a set of selected instruments. As a follow on of PROBA1 [2] successfully in orbit since October 2001, the performances and the autonomous functions previously demonstrated by PROBA-1 will also be met or exceeded.

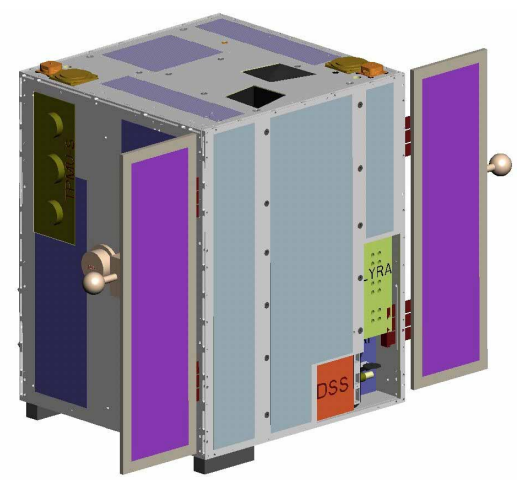

Fig. 1. View of the PROBA-2 platform (Sun side)

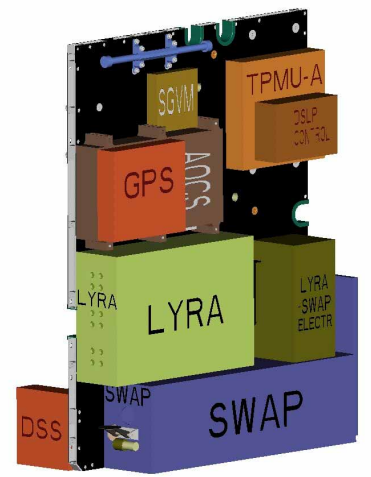

Fig. 2. View of the PROBA-2 payload

The technology demonstrations are in the field of avionics, spacecraft attitude control, power system and spacecraft propulsion. Two instruments are dedicated to the observation of the Sun, SWAP (imaging instrument) and LYRA (radiometer instrument), and 
two others are dedicated to plasma measurements. Possibly an Earth observation instrument will be included as well.

\section{SWAP: A SCIENCE INSTRUMENT}

SWAP has been proposed as a kind of successor of the Extreme ultraviolet Imaging Telescope [1] (EIT) onboard the joint ESA-NASA mission SOHO. The latter monitors the solar corona since 1996. In its nominal 'CME watch program', EIT takes an image in its $19.5 \mathrm{~nm}$ bandpass every $15 \mathrm{~min}$. The EIT instrument has proved to be particularly useful for space weather monitoring.

The $19.5 \mathrm{~nm}$ bandpass that SWAP will inherit from EIT gives indeed a particularly broad view on the 'solar weather'. Single $19.5 \mathrm{~nm}$ images already give important information on the location of coronal holes (the source of high speed solar wind streams), active regions (potential source of solar flares) and filaments (potential eruption sites). Following the temporal evolution of these features as they rotate, gives additional important inputs to space weather forecasters.

Yet, accurate monitoring is only achieved by having high cadence time sequence of $19.5 \mathrm{~nm}$ images. Such sequences give information on more subtle events like EIT waves [3], coronal EUV dimming regions and filament instabilities. Systematic detection by the SWAP instrument of these phenomena that are associated with the early development of CMEs allows to pinpoint the source region of coronal mass ejections and let differentiate between back-sided and frontsided halo CMEs. The accurate identification of the last type of CMEs gives a 3-day warning before the CME can hit the Earth magnetosphere and cause geomagnetic storms.

SWAP will continue the systematic CME watch program of the ageing EIT instrument, but at an image cadence (1 image every 2 minutes or faster, instead of every 15 minutes) that is more adequate to its spatial resolution (2.6 arcsec). Most space weather significant events last on the order of $30 \mathrm{~min}$ to $45 \mathrm{~min}$ : EIT waves $(45 \mathrm{~min})$, prominence eruptions $(30 \mathrm{~min})$ and flares (M-flare: $24 \mathrm{~min}$, X-flare: $30 \mathrm{~min}$ ) [4]. This means that the EIT CME watch was sufficient to record the occurrence of these events, but not their temperature evolution (in the case of EIT waves or eruptions), or peak emission (in the case of flares). Thanks to this higher image cadence, SWAP will be a solar monitor capable of recording the time-evolution of every event in the low solar corona that might be of relevance to the space weather.
In addition to space weather monitoring, SWAP will also be used for basic solar research, especially when used in joint observation campaigns with other missions. The EUV imagers of the NASA STEREO/SECCHI [5] mission will have a similar spectral bandpass at $19.5 \mathrm{~nm}$, making SWAP a potential third eye of this 2 -spacecraft mission.

\section{SWAP: A NEW EUV TELESCOPE}

\subsection{Instrument Concept}

SWAP will provide 1-Mpixel images of the EUV solar corona at $19.5 \mathrm{~nm}$ in a square field of view of 45 arcmin. It will use an off-axis telescope derived from a Ritchey-Chretien system. An elliptical primary mirror combined with a spherical secondary mirror used in quasi-normal incidence will focus the solar light on a detector implemented in a focal plane assembly. The overall optical elements are mounted on an optical bench to provide the necessary stability. The spectral selection is achieved with a set of aluminium foil filters, together with EUV reflective multilayer coatings deposited on the mirrors. The detector is a CMOS device coated with a scintillator layer to improve sensitivity in the EUV range. It is passively cooled with an external radiator viewing cold space to reduce the thermal noise. This type of detector has advantages that promise to be very profitable for solar EUV imaging from space such as radiation resistance, shutterless operation, non destructive readout ...

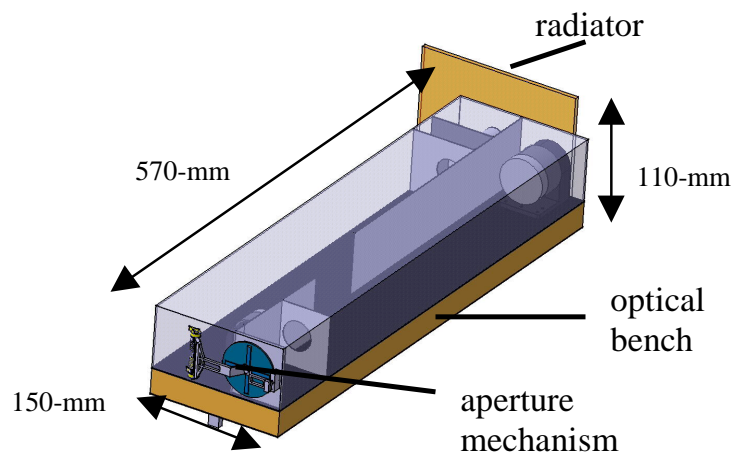

Fig. 3. SWAP sensor box

The instrument is encased in a lightweight housing equipped with an aperture mechanism to preserve the optical cavity from external contamination during onground activities and launch phase. SWAP is mounted on the spacecraft platform with a set of isostatic flexible mounts.

The sensor box includes its proximity electronics for readout, while the dedicated instrument electronics boards are embedded in the central computer of the PROBA-2 spacecraft. 


\subsection{Optical Scheme}

The optical scheme is designed upon a 2-mirror offaxis system. This concept offers a good optical quality in a minimum length, and the off-axis configuration allows small mirrors, by avoiding central obstruction, and consecutively reduces the aluminium filter foil size. Moreover this configuration allows a simple baffling system without requiring any external front baffle, which would not be compliant with the limited allocated volume on the platform.

The basic optical requirements are summarised in table 1. The field of view is defined to adequately cover the solar corona according to the scientific needs, while spacecraft accommodation restrictions dictate the total allowable length. The entrance pupil diameter is the result of a trade off between several parameters:

- available off-the-shelf aluminium filters (restrictions on diameters),

- $\quad$ limit the filter size to reduce risks of damage (air flow and acoustic pressure during launch),

- $\quad$ keep a sufficient optical throughput,

- lateral extension of the off-axis folding due to the physical size of the mirrors and limitation of the incidence angles to keep a good coating reflectivity.

The narrow spectral range is imposed by the science use of the instrument. It is centred on a bright emission line (Fe XII) of the EUV solar corona, with a limited bandwidth to avoid crosstalk with other emission lines.

\begin{tabular}{|c|c|}
\hline Field of view: & 45 arcmin square \\
\hline $\begin{array}{c}\text { Spectral } \\
\text { wavelength: }\end{array}$ & $\begin{array}{c}19.5 \mathrm{~nm} \text { central wavelength } \\
(1 \mathrm{~nm} \text { FWHM bandpass })\end{array}$ \\
\hline Total length & $<450 \mathrm{~mm}$ \\
\hline Max width & $33 \mathrm{~mm}$ diameter \\
\hline Entrance pupil: & $1173.35 \mathrm{~mm}$ \\
\hline Focal length: & $\begin{array}{c}\text { CMOS STAR1000 from } \\
\text { Fillfactory }[6]\end{array}$ \\
\hline Detectors & $1024 \times 1024$ pixels $(15 \mu \mathrm{m}$ pitch $)$ \\
\hline
\end{tabular}

Table 1. Basic optical requirements

The optical layout is shown in Fig. 4. The entrance pupil is located at the front side of the instrument, in close vicinity with the aluminium filter. This configuration provides the smallest filter section for a given aperture, as it is required to avoid it in the double pass region, closer to the primary mirror.

The primary mirror is elliptical (44 $\mathrm{mm}$ diam.), while the secondary mirror (28 mm diam.) is kept spherical for the sake of simplicity, as SWAP is developed under extreme schedule constrains.

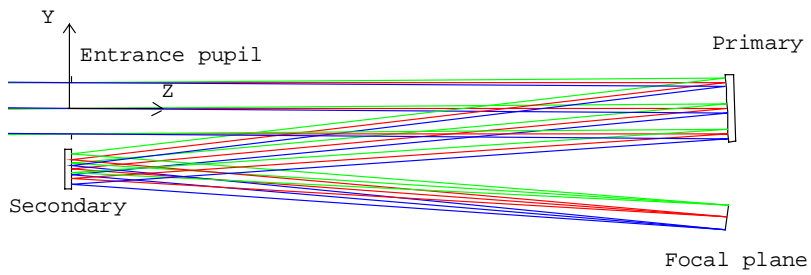

Fig. 4. Optical layout of SWAP

The optics are tilted to compensate the remaining aberrations. The paraxial focal length is adjusted to take into account distortion and fit with the field of view.

\subsection{Optical Performances}

By design, the RMS spot diameter varies between 1.6 $\mu \mathrm{m} \quad(0.28$ arcsec $)$ and $10.6 \mu \mathrm{m} \quad(1.87$ arcsec $)$ respectively for an on-axis object and for the corners of the field of view. The design is mainly limited by astigmatism induced by the spherical secondary mirror. The distortion remains lower than $0.5 \%$. The performances are illustrated in Fig. 5 and 6.

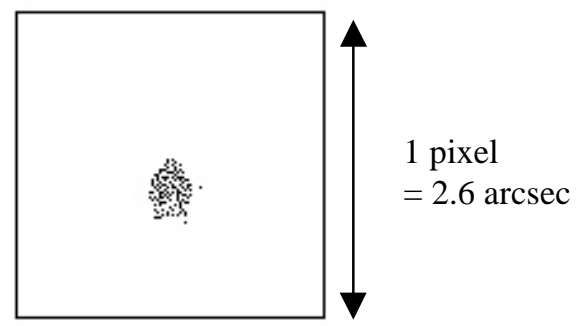

Fig. 5. RMS spot on axis
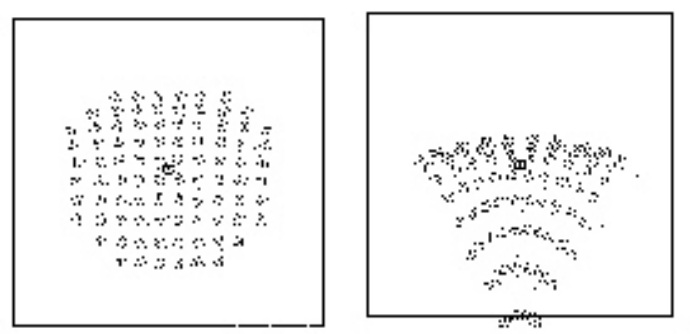

Fig. 6. RMS spot size at borders of field of view (22.5 arcmin)

A manufacturing and alignment budget has been derived to account for all the contributions that will affect the resulting optical quality (see table 2). It results into a $22.5 \mu \mathrm{m}$ RMS spot diameter (3.95 $\operatorname{arcsec}$ ). The main contributions come from the in-flight conditions (mainly the distance primary - secondary mirrors) and the spacecraft jitter $( \pm 3$ arcsec in the spectra $0.2-10 \mathrm{~Hz})$ during the integration time $(60$ sec). The first contribution affects highly the thermomechanical design of the optical bench. 
Superpolished optics are required to limit the EUV light diffusion by mirrors optical surfaces that spreads the PSF and decreases the resolution. A microroughness below $0.5 \mathrm{~nm}$ is required, which is common but still a challenge, for EUV imaging optics.

\begin{tabular}{|c|c|}
\hline Contributions & $\begin{array}{c}\text { RMS spot size } \\
\text { degradation } \\
\text { (at 16 arcmin FOV) }\end{array}$ \\
\hline Design & $7.5 \mu \mathrm{m}$ \\
\hline Mirror figuring & $0.2 \mu \mathrm{m}$ \\
\hline Mirror irregularities & $13 \mu \mathrm{m}$ \\
\hline Alignment & $1.16 \mu \mathrm{m}$ \\
\hline Launch effect & $0.05 \mu \mathrm{m}$ \\
\hline In-flight conditions & $3.6 \mu \mathrm{m}$ \\
\hline Spacecraft pointing & $6.46 \mu \mathrm{m}$ \\
\hline Total & $\mathbf{2 2 . 5} \boldsymbol{\mu m}$ \\
\hline
\end{tabular}

Table 2. Manufacturing and alignment budgets

\subsection{EUV Reflective Coatings}

The mirrors of SWAP will be treated with a specific periodic multilayer coating. This interferential filter has to provide adequate spectral selection, as well as a maximum reflectivity at the wavelength of interest.

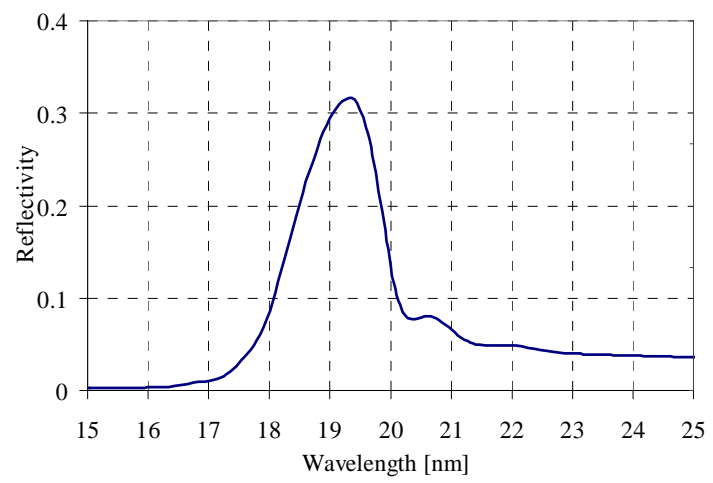

Fig. 7. Typical EUV reflectivity of a $19.5 \mathrm{~nm}$ optimized coating (single reflection)

The SWAP EUV coatings will be similar to those developed for the EIT-SOHO [7] and the STEREO SECCHI [5] programs.

\subsection{Optical Baffles}

A baffling structure needs to be implemented to avoid direct illumination of the detector. This off-axis scheme allows a simple and efficient baffling limited to the inter-mirror volume, without need to be extended outside the instrument, and without stringent positioning tolerances. Two planar baffles have been designed, according to the scheme shown in Fig. 8.

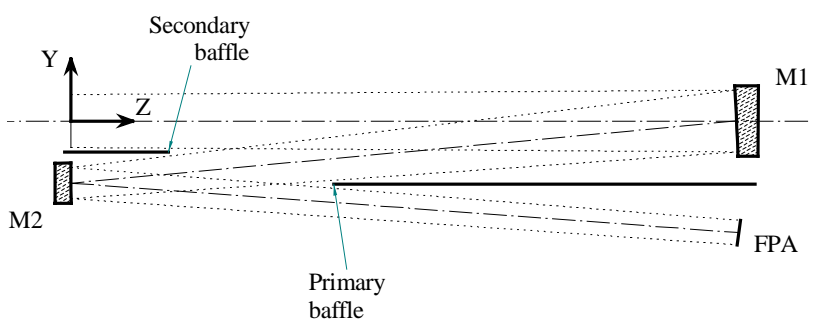

Fig. 8. Optical cavity with baffles

\section{CRITICAL DESIGN ISSUES}

\subsection{Thermal issue}

SWAP is mounted on an uncontrolled platform, originally designed for technological experiments without stringent stability requirements. Therefore SWAP has to account for a wide thermal range, amplified with the periodic eclipses of the PROBA-2 orbit. On the other hand, SWAP has very limited power resource without any allocation for an active thermal control system. Therefore, optomechanical concept must be able to passively keep its performance over the required thermal range.

A sensitivity analysis has been run to evaluate the effects of thermal expansion of the bench supporting the optics. The degradation of the $70 \%$ encircled energy diameter is of the order of $0.016 \mathrm{arcsec} / \mu \mathrm{m}$ of M1-M2 variation, for a pixel platescale of 2.6 arcsec/pixel (see Fig. 9).

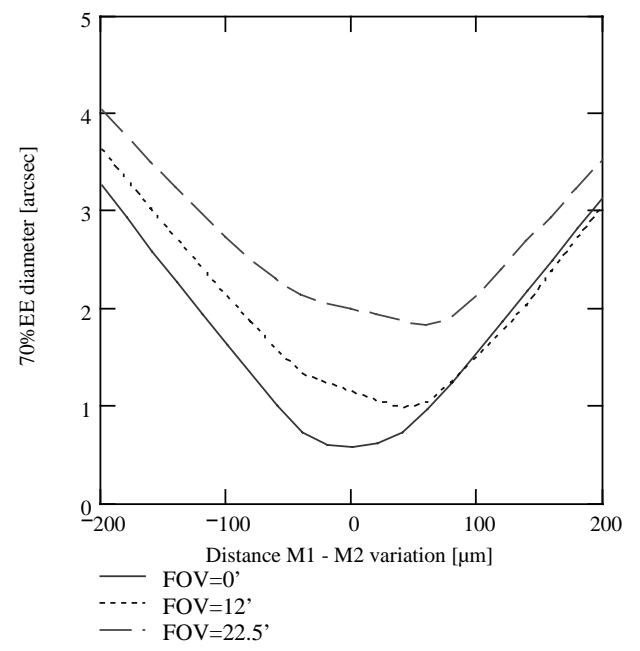

Fig. 9. Degradation of the $70 \%$ encircled energy diameter with inter-mirror distance variations 
Those results can finally be converted in effective spot degradation for different optical bench materials submitted to a given temperature variation (see table $3)$.

\begin{tabular}{|l|c|}
\hline $\begin{array}{l}\text { Optical bench with } \\
10^{\circ} \mathrm{C} \text { variation }\end{array}$ & $\begin{array}{c}\text { Spot degradation } \\
\text { (diameter) }\end{array}$ \\
\hline Aluminium alloy & 1.55 arcsec \\
\hline Titanium alloy & 0.53 arcsec \\
\hline INVAR & 0.08 arcsec \\
\hline
\end{tabular}

Table 3. Thermal effects on the $70 \%$ encircled energy diameter

The SWAP optical bench has been designed based on these considerations. An INVAR structure is under development to accommodate the optics, while keeping the overall mass budget below $9 \mathrm{~kg}$ for the sensor box, without active thermal control.

A premiminary view of the SWAP Invar structure is shown in Fig. 10 (courtesy AMOS S.A.).

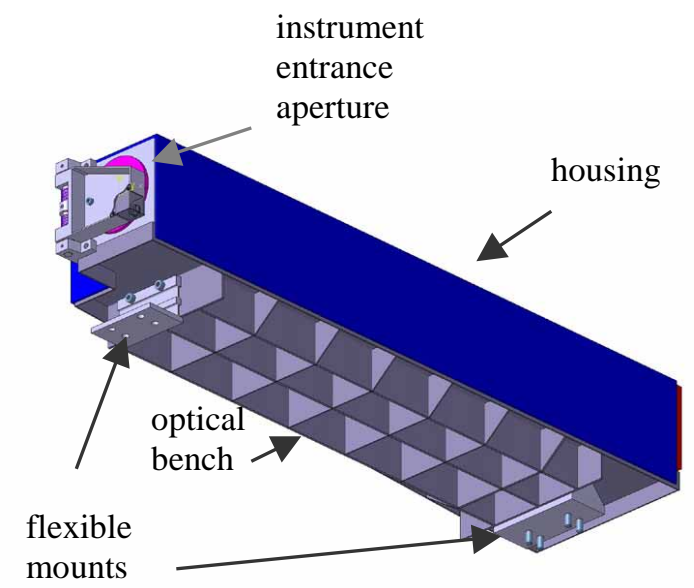

Fig. 10. Preliminary view of the optical bench with its housing (bottom view)

\subsection{Optical artefacts of filter support grids}

The observation of the low EUV radiance of the Sun at $19.5 \mathrm{~nm}$ requires a very high rejection of the visible and UV solar flux, with rejection rates of the order of $10^{-8}$. To achieve this performance, aluminium offers a good transmission spectrum, with a high reflectivity in the visible, and a transmission window in the range of 17 to $80 \mathrm{~nm}$, as illustrated in Fig. 11. SWAP includes 2 foil filters, one at the instrument entrance to avoid excessive heating of the primary mirrors, and one near the focal plane to eliminate any residual visible straylight from potential light leaks in the front housing or the entrance filter itself.

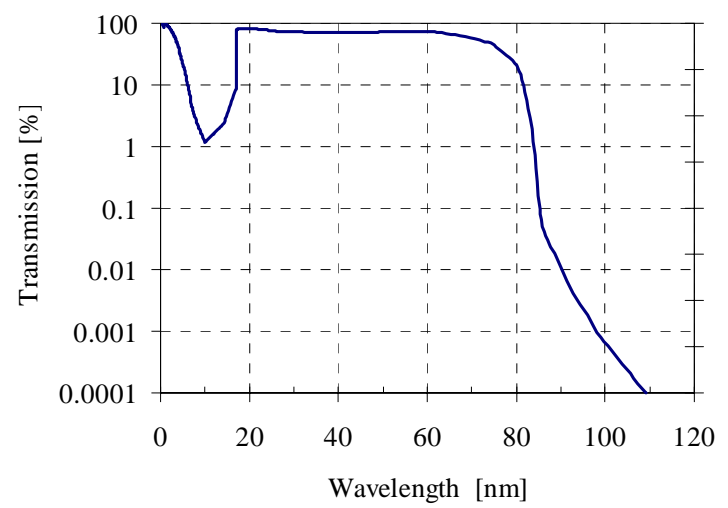

Fig. 11. Typical transmission of a single aluminium foil filter (150-nm thickness)

Self-standing aluminium foil filters of $150 \mathrm{~nm}$ thickness and $40 \mathrm{~mm}$ diameters cannot reasonably survive the mechanical and acoustic environment during the launch. Therefore, the aluminium foil is supported by a metallic opaque grid. Commercial filters are available with grid period of 70 lines/inch, with $38 \mu \mathrm{m}$ diameter opaque wires. This corresponds to a transparency of $80 \%$. Such a grid interacts with the optical beams in different ways, depending on their location.

The entrance filter is nearby the entrance pupil, it will not be imaged, but it will induce diffraction effects in the focal plane. A simulation has been run, using Fresnel propagation laws applied to the pupil masked with the opaque grid. The results are shown in Fig. 12, in which the pixel discretization and the 12-bit scaling are taken into account. The diffraction perturbation will only be effective locally in very bright regions, while it will be undetectable on most of the image area. If needed, deconvolution will be used to remove this artefact.

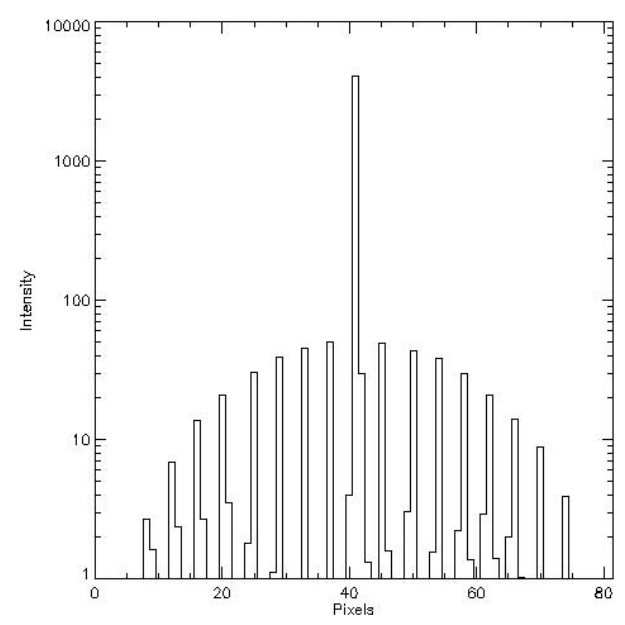

Fig. 12. PSF affected by the diffraction of the grid 
The focal filter is nearby the focal plane. The dominating perturbation is a shadowing effect that produces a periodic modulation. Simulations have been run to derive a shadow map corresponding to a 70 lines/inch grid located at $35 \mathrm{~mm}$ from the detector. As already studied and processed with the EIT solar images affected by the same perturbations [8], this constant shadowing can be mapped and used for correction. This post-processing is planned for SWAP images.

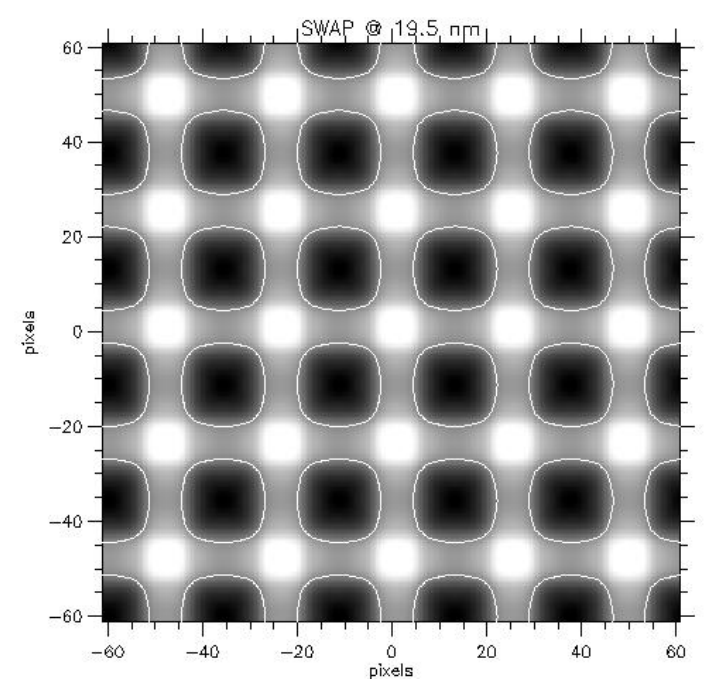

Fig. 13. Modulation pattern due to the focal filter grid on a $120 \times 120$ pixels area (peak-topeak variation $\sim 12 \%$, mid-range is outlined with white contour)

\section{CONCLUSIONS}

SWAP is a science instrument with several technological challenges. However, it is built upon proofed heritage, that should ensure that SWAP will be an effective and efficient space weather tool.

The opportunity offered by the PROBA-2 technological platform has been taken to provide one side science data, and on another side, to demonstrate several new concepts such as a new off-axis EUV optical scheme, the use of a CMOS detector for EUV imaging in space, a lightweight INVAR optical bench, and on-board data processing.

\section{ACKNOWLEDGMENTS}

The development of the SWAP instrument is funded by the Belgian Federal Science Policy Office, through the ESA/PRODEX programme.

We wish to thank the MPAe team for the support provided for the preliminary EUV measurements on the CMOS detectors.

\section{REFERENCES}

1. JP. Delaboudinière, G.E. Artzner, J. Brunaud, A.H. Gabriel, J.F. Hochedez, F. Millier, X.Y. Song, B. Au, K.P. Dere, R.A. Howard, R. Kreplin, D. J. Michels, J.D. Moses, J.M. Defise, C. Jamar, P. Rochus, J.P. Chauvineau, J.P. Marioge, R.C. Catura, J.R. Lemen, L. Shing, R.A. Stern, J.B. Gurman, W.M. Neupert, A. Maucherat, F. Clette, P. Cugnon, E.L. Van Dessel., EIT: Extreme-UV imaging telescope for the SOHO mission; Solar Physics 162: 291-312, 1995.

2. F. Teston, R. Creasey, J. Bermyn, K. Mellab, "PROBA: ESA's Autonomy and Technology Demonstration Mission", Proceedings of the 13th Annual AIAA/USU, Conference on Small Satellites, August 23 - 26, 1999.

3. Thompson, B. J.; Plunkett, S. P.; Gurman, J. B.; Newmark, J. S.; St. Cyr, O. C.; Michels, D. J. SOHO/EIT observations of an Earth-directed coronal mass ejection on May 12, 1997. GRL 25, No. 14, p. 2465 - 2468 (1998).

4. Veroniq, A. et al, "Temporal aspects and frequency distributions of solar soft X-ray flares"“,AA, 382, 1070, 2002.

5. J.D. Moses, R.A. Howard, K.P. Dere, D. Socker, J. Karpen, J. Klimchuk, N. Sheely, C. Korendyke, D. Michels, M. Koomen, O.C. St Cyr, S. Plunkett, A. Title, J.P. Wuelser, J. Lemen, T. Tarbell, C. Wolfson, M. Aschwanden, D. Alexander, T. Metcalf, J. Davila, R. Fisher, B. Thompson, J. Gurman, J. Newmark, L. Burlaga, L. Golub, P. Liewer, E. De Jong, Z. Mikic, J. Linker, D. Hassler, L. Fisk, T. Zurbuchen, G. Simnett, L. Culhane, R. Harrison, R. Bush, R. Schwenn, B. Inhester, E. Marsch, V. Bothmer, H. Kunow, J.P. Delaboudinière, B. Agius, R. Mercier, J.L. Bougeret, M. Pick, P. Lamy, A. Llebaria, J.M. Defise, C. Jamar, P. Rochus, D. Berghmans, F. Clette, J.F. Hochedez, P. Cugnon, Sun-Earth connection coronal and heliospheric investigation (SECCHI) for the NASA STEREO mission, SPIE 4139-26, Jul 2000.

6. B. Dierickx, G. Meynants, D. Scheffer, "Near 100\% fill factor CMOS active pixels", in IEEE CCD \& AIS workshop, Brugge, Belgium, 5-7 june (1997); Proceedings p. P1

7. JP Chauvineau et al, "Description and performances of mirrors and multilayers for the extreme ultraviolet imaging telescope (EIT) of the SOHO mission", Proc. SPIE 1546, 576.

8. J.M. Defise; F. Clette, F. Auchere, In-flight characterization and compensation of the optical properties of the EIT instrument, SPIE 3765, 1999. 